\title{
Priority of Treatment Outcomes for Caregivers and Patients with Mild Cognitive Impairment: Preliminary Analyses
}

\author{
Polaris González Barrios • Ricardo Pabón González • \\ Sherrie M. Hanna · Angela M. Lunde · Julie A. Fields · \\ Dona E. C. Locke · Glenn E. Smith (D)
}

Received: June 2, 2016 / Published online: August 9, 2016

(C) The Author(s) 2016. This article is published with open access at Springerlink.com

\begin{abstract}
Introduction: The patient-centered movement advocates for greater attention to the outcomes that matter most to patients and their families. In neurodegenerative disease, determination of patient and caregiver priorities has received scant attention in part because dementia patients are deemed unreliable reporters. However, people
\end{abstract}

Enhanced content To view enhanced content for this article go to www.medengine.com/Redeem/ 3AE4F0607B87DD23.

Electronic supplementary material The online version of this article (doi:10.1007/s40120-016-0049-1) contains supplementary material, which is available to authorized users.

P. G. Barrios · R. P. González

Ponce School of Medicine \& Health Sciences, Ponce, Puerto Rico

S. M. Hanna · A. M. Lunde · J. A. Fields Division of Neurocognitive Disorders, Department of Psychiatry \& Psychology, Mayo Clinic, Rochester, MN, USA

D. E. C. Locke

Department of Psychiatry \& Psychology, Mayo

Clinic, Scottsdale, AZ, USA

\section{G. E. Smith (ه)}

Department of Clinical and Health Psychology,

University of Florida, Gainesville, USA

e-mail: glennsmith@phhp.ufl.edu with mild cognitive impairment (MCI) likely retain capacity to report their preferences.

Methods: In two separate MCI cohorts, we conducted preliminary analyses of patient and caregiver priorities among seven patient and five caregiver outcomes of the HABIT ${ }^{\circledR}$ Healthy Action to Benefit Independence \& Thinking program (Mayo Clinic, Rochester, MN, USA).

Results: Via interview and paper-and-pencil reporting both patient and caregiver respondents' ranked patient and caregiver quality of life and patient self-efficacy as highest priorities, ranking them ahead of patient and caregiver mood, patient functional status, patient distressing behaviors and caregiver burden. Patients and caregivers tended to value the outcomes for their loved ones higher than their own outcomes.

Conclusion: Caregivers appeared to be reasonable, but not perfect, proxies for patient reports. Additional research with larger cohorts and a more comprehensive range of outcomes is needed.

Keywords: Alzheimer's disease; Caregivers; Mild cognitive impairment; Patient preference; Quality of life 


\section{INTRODUCTION}

The patient-centered outcomes research movement has been galvanized and promoted by the Patient-Centered Outcomes Research Institute. Patient-centered outcomes research includes helping people and their caregivers consider their full range of healthcare options in terms of the risk and benefits. Patient centeredness promotes patients and caregivers making and communicating healthcare decisions informed by their personal characteristics, conditions, and preferences. One set of key preferences involves specifying outcomes that matter most to patients and caregivers [1].

Bringing patient centeredness to care and research in the area of central nervous system diseases, including neurodegenerative diseases, is complicated by concerns about patients' capacity to state their preferences in a fully informed fashion [2]. For this reason, some have argued that using patient and caregiver-reported outcomes, such as quality of life (QoL) [3] measures, is ill-suited to research in Alzheimer's disease (AD) or related conditions [4]. However, modern nomenclature of neurodegenerative disease diagnosis recognizes an initial phase of the illness characterized by concerning, but mild cognitive impairments (MCI) with retained functional capacities. These retained functional capacities enable an individual to establish health care preferences and participate in health care decision-making [5]. This phase of illness is labeled MCI [6-8] or mild neurocognitive disorder [9]. Patients with MCI are at high risk to progress to dementia, most often related to AD. But patients with MCI do not yet have the impairments in medical decision-making capacity seen in patients with mild dementia due to AD [5]. This status affords a special opportunity to consider outcome preferences for patients and their caregivers, including the opportunity to determine whether patient and caregiver preferences are generally consistent across the couple and whether they change with clinical intervention.

In the present pair of preliminary studies, our aim was to explore the most important behavioral outcomes for caregivers and patients with MCI.

\section{METHODS}

We used convenience cohorts assembled from the HABIT $^{\circledR}$ Healthy Action to Benefit Independence and Thinking program (Mayo Clinic, Rochester, MN [10]). HABIT is a multi-component behavioral intervention program for persons with MCI and their partners. In the HABIT program, the patients are referred to as the participants. The caregivers are referred as 'partners' because in many case they are not yet providing much care. Patient/participant and caregiver/partner labels are used interchangeably herein. Data collection for this program focuses on an array of patient- and caregiver-reported outcome measures (see Table 1). Patients and caregivers complete the measures listed in Table 1 just before, immediately after and at one-year intervals following the HABIT program. They are thus familiar with the constructs we were asking them to prioritize, at least as measured by these instruments. This gave them the opportunity to associate the outcomes we were asking them to rank (e.g., patient's memory-based daily function) to a specific measure (The Everyday Cognition scale, [11]) that the patients or their caregivers had previously completed. 


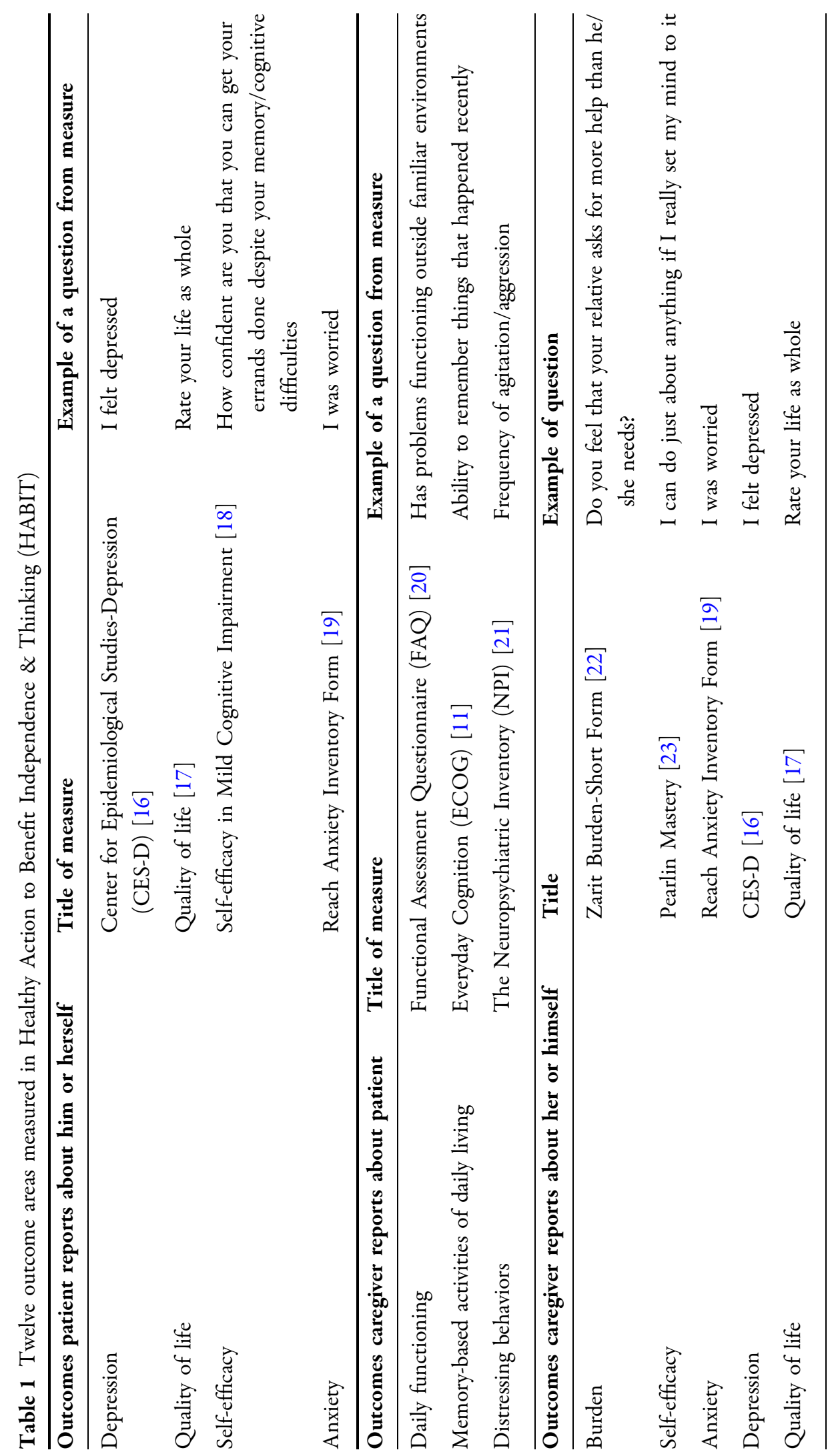


The first study was intended to pilot our forced-choice ranking method, so we limited the cohort to cognitively intact caregivers that had previously completed the HABIT program with their participants who had MCI. These caregivers were completing the outcome measures as part of on-going longitudinal follow-up of program outcomes. The caregivers prioritized the importance of the various constructs the program evaluation measures were intended to assess. This analysis had the additional benefit of addressing the problem that caregivers' preferences are generally not considered when addressing priorities for, and impacts of, treatments for dementia [12]. In the second study, we sought to obtain the perspective of the patient him or herself, examine the concordance between patient and caregiver rankings, and to explore whether there were shifts in reported priorities from pre- to post-treatment. In the second study, patients with MCI and their respective caregivers (different from the first study) were surveyed. In study two, the patients and the caregivers were surveyed immediately before and immediately after completion of the HABIT program. In both studies data were stored and statistics performed using Microsoft Excel for Mac Version 14.5 (Microsoft Corp, Seattle, Washington, USA).

\section{Study 1}

\section{Participants}

Participants in the HABIT program are generally referred from the Behavioral Neurology or Neuropsychology practices at the Mayo Clinic though couples whom learn of the program via the internet can be self-referred. Patients are required to have a medical diagnosis of MCI. Patients generally met standard Mayo diagnostic criteria for MCI $[7,8]$. Prior to initiating the program cognitive status is verified by administration of Dementia Rating Scale (DRS) [13]. Patients must score $>115$ on the DRS to participate in the program. All 36 caregiver alumni that completed the Mayo HABIT program at Mayo Clinic Minnesota or May Clinic Arizona during calendar year 2012 were candidates for the study. Three could not be reached by phone. The remaining 33 contacted via telephone consented to participate in in-person or telephone interviews to follow-up on perceived outcomes from the program. All caregivers had previously completed the set of 12 patient/caregiver-centered outcomes measures (See Table 1) routinely used to assess HABIT clinical outcomes and evaluate the HABIT program. Caregivers for the HABIT program are required to have a Mini Mental State Examination (MMSE) scores in the normal range ( $\geq 27$ ) and to have regular (at least once weekly) contact with the person with MCI.

\section{Interview}

The primary author obtained consent and completed a structured interview with all caregivers. In the interview, she used the method of pairwise comparisons for rank ordering on a scale of $1=$ most important to 12 = least important (see S1). It typically required more than 30 paired comparisons to determine the rank order.

\section{Study 2}

\section{Participants}

Couples attending the summer 2014 HABIT sessions at Mayo Clinic Rochester agreed to participate in the study. Rank prioritization was again on scale from $1=$ most important to 12 = least important. However, for this study data were collected via paper forms rather than 
in-person interview (see S2). Inclusion and Exclusion criteria were the same as study 1 . Again all patients were required to have a caregiver with at least once weekly contact with the patient and MMSE $\geq 27$.

\section{Pre and post-intervention rankings}

Rankings were made no more than 1 month prior to beginning of the 10-day program. Participants and partners then completed the five components of the program totaling $50 \mathrm{~h}$ of intervention: (1) Memory compensation training, (2) Yoga, (3) Computerized cognitive stimulation, (4) Wellness education, (5) Support group therapy. Patients and caregivers completed the rank ordering of the 12 outcomes again on the last day of the program.

\section{Compliance with Ethics}

These studies were approved by the Mayo Clinic Institutional Review Board. All procedures followed were in accordance with the ethical standards of the responsible committee on human experimentation (institutional and national) and with the Helsinki Declaration of 1964, as revised in 2013. Informed consent was obtained from all patients for being included in the study.

\section{RESULTS}

\section{Study 1}

Partner respondents were $91 \%$ female with a mean age of 71 years and $54 \%$ were college graduates. In this analysis, $87 \%$ of caregivers were spouses and the remainder were adult children of the person with MCI. The results of the rank ordering of outcome priorities of caregivers of MCI patients are depicted in
Fig. 1. The patient's QoL has the highest average ranking and was endorsed as the most important treatment outcome by $30 \%$ of caregivers. Patients' self-efficacy in handling their MCI ranked second but was comparable to patient QoL. Of note, caregivers did value their own QoL but less than they valued the patients' QoL $(p<.05)$. The patients' daily functioning on memory-based activities was ranked in the upper half of outcomes but not as highly as QoL outcomes. Caregiver depression and burden were of least importance among all treatment outcomes, and ranked last by more than $25 \%$ of caregivers.

\section{Study 2}

All 16 couples attending the HABIT session agreed to participate. Seventy-five percent of the patient respondents were male. The mean (standard deviation) age of patients was 77.3 (7.1) and mean education was 16.3 (2.5). Caregivers were $81 \%$ female. Twelve of the caregivers were spouses, and three more were unmarried romantic partners. One caregiver was a friend. Mean age of the caregiver group was 73.1(7.5) and mean education was 14.9(1.5) years. The pre- and post-intervention average rankings for MCI patient and caregivers are presented in Fig. 2.

\section{Pre-program Rankings}

On average patients ranked their own QoL and self-efficacy first and second respectively, followed by caregiver QoL and caregiver mood. Distressing behaviors were least concerning to the patients. Caregivers ranked patient QoL, patient mood, and caregiver QoL as the top three important outcomes, respectively. Fifty percent of caregivers ranked patient QoL as their top priority. 


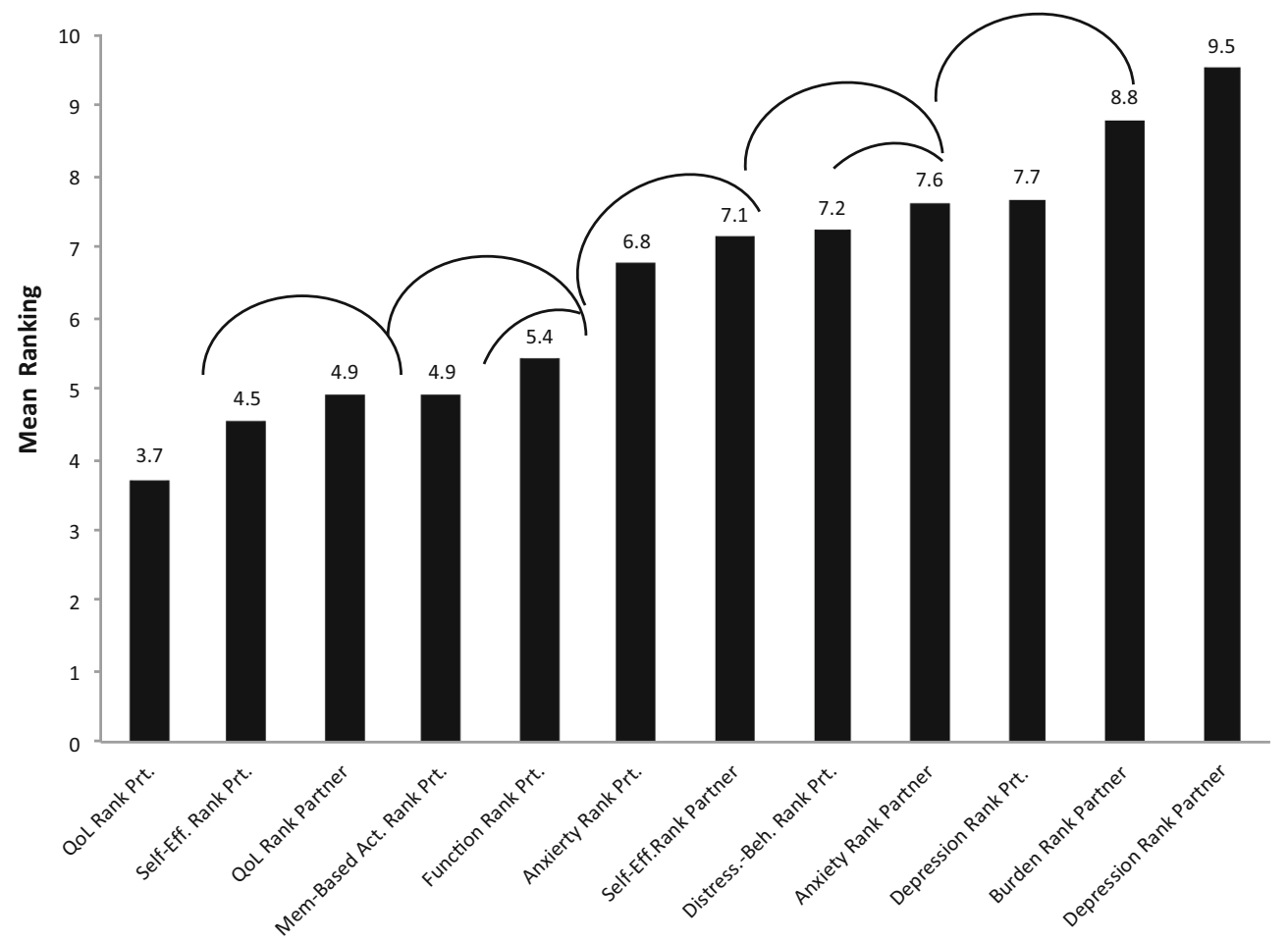

Fig. 1 Mean rankings of post-intervention outcome priorities for caregivers (study 1). Rank ordering on a scale of $1=$ most important to $12=$ least important thus lower rankings equal higher priority. Arcs identify rankings

\section{Post-program Rankings}

Dependent $t$ tests showed a trend for patients to have changed their prioritization of caregiver burden after participation in the HABIT program. This outcome moved from an average ranking of 6.5 to an average of 4.3 $(p=.09)$ suggesting increased concern about their partner's burden after participation in the program As a result, on average, it moved from sixth highest outcome to the highest ranking. No other changes approached statistical significance. None of the caregiver rankings changed significantly from pre- to post-intervention. There was one trend observed. Patient depression trended toward lower priority for caregivers, moving from the second highest to the sixth highest average rank $(p=.06)$. This suggests that caregivers became that differ at $p<.05$. Beh, behavior; Mem-Based Act, memory-based activities of daily living; $Q o L$, quality of life; Prt, participant; Self-Eff, self-efficacy

less concerned about patient's mood outcomes from baseline to end of program.

\section{Patient Versus Caregiver Ranking}

In comparing patient and caregiver pre-program average rankings only one significant difference emerged. Caregivers appeared more concerned about patient depression than were the patients (mean caregiver rank 4.2, mean patient rank $7.9, p<.01)$. Conversely there was a trend for patients to be more concerned about caregiver burden than are the caregivers themselves (mean patient rank 6.2, mean caregiver rank $8.9, p=.08)$. However, patients and caregivers agree the most important outcomes involve QoL of patients, and that distressing behaviors (hallucinations, delusions, agitation) are one of the least important (at the MCI stage). 


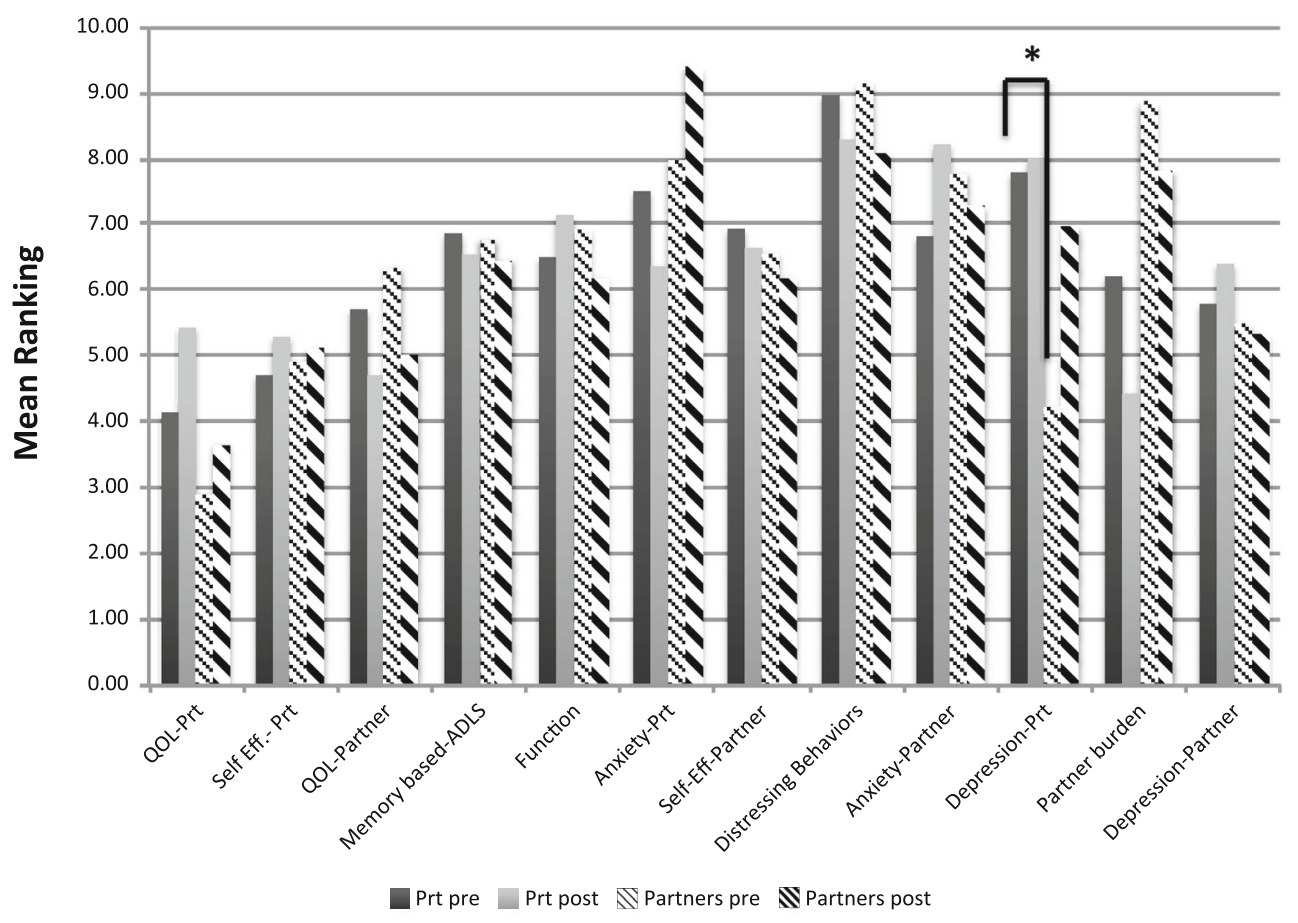

Fig. 2 Pre- and post-intervention rankings for patients with MCI and their caregivers (study 2). Rank ordering on a scale of $1=$ most important to $12=$ least important thus lower rankings equal higher priority. Asterisked line

\section{DISCUSSION}

We examined the relative importance of a variety of patient-reported and caregiver-reported outcomes to patients and caregivers that were reporting on these outcomes. The outcomes for patients included basic and memory-based daily function, anxiety, depression, distressing behaviors, self-efficacy, and QoL. We also inquired about caregiver outcomes including burden, depression, anxiety, self-efficacy, and QoL. The present results suggest that on average at the MCI stage, patient QoL and self-efficacy are most important to both patients with MCI and caregivers. These priorities seemed to hold across methods (face-to-face or phone interview, paper-and-pencil survey). Concerns about more traditional outcomes of anxiety, identifies rankings that differ at $p<.01 . A D L S$, activities of daily living; QoL, quality of life; Prt, participant; Self-Eff, self-efficacy

depression, and daily function were not as highly prioritized. These priorities may emerge in later stages of neurodegenerative disease [14]. At the MCI stage, the caregivers and patients seeking an intensive multi-component behavioral intervention are focused on patients' opportunities to gain skills that may sustain perceived QoL and enhance self-efficacy regarding their ability to manage memory impairment.

The results also show that on average the high prioritization of QoL remains after an intensive behavioral intervention. However, the HABIT intervention completed by these patients seemed to sensitize them to the needs of their caregivers, causing their priority for caregiver burden in particular, to rise to a top concern post-treatment. Because we permitted patients and caregivers to rate both patient and 
caregiver outcomes, we seemed to observe a 'gift of the Magi' effect where patients and caregivers prioritized their partners outcomes over their own. Participation in the intervention program seemed to amplify this effect. Permitting patients and caregivers to prioritize their partners' as well as their own outcomes may have led to lower estimates of concordance in this study relative to others (cf. [15]).

This analysis is limited in several respects. First, it focuses only the limited set of outcomes used in our intervention program. We chose to do this because we believed the completion of the outcome measures for the program made the respondents far more familiar with the constructs they were ranking. Had they only been prioritizing the constructs based on their labels (e.g., 'memory-based daily functions') there might have been more challenges understanding what the terms meant. Nevertheless, we acknowledge that a variety of traditional clinical trial outcomes including actual cognitive function and a range of biomarkers (brain volumes, amyloid levels, etc.) were not subjected to ranking. Including these traditional outcomes in future examinations of patient and caregiver outcome preferences could be a focus of future analysis.

Second, our method of rank ordering places certain constraints on how these outcomes may relate to each other. We chose this method in deference to other approaches (e.g., likert scaling) as it was very easy for participants to understand. We acknowledge that rank ordering meant different outcomes could not have equal importance to one another. All outcomes were forced to be either more or less important than those of proximal ranking. Patients and caregivers may actually perceive some of these outcomes to be equally important (or unimportant). This approach could have served to magnify differences in the rankings.
Conversely, the rank ordering system limited the degree to which proximal items could differ, i.e., by only one rank unit. For example, a person could not declare that his or her highest ranked item was far more important than the second ranked item, which was only slightly more important than the third ranked item. Thus, the rank ordering may have served to diminish the differences in how these outcomes are actually valued. In any event, future studies using different methods for determining patient and caregiver outcome preferences are needed to determine the reliability of the present findings.

A final limitation is the nature of the cohorts used in these studies. They were small convenience samples. A larger sample would have provided more power to identify statistical differences between outcomes, groups, subgroups, or time points. Moreover, the sample is clearly limited in ethnic and educational diversity. It is comprised of people motivated to participate in an intensive multi-component behavioral intervention. This selection factor alone likely biases how different outcomes are viewed. However, from a PCOR research perspective the preferences of this group are exactly the preferences to identify. That is, it is a key to understand the preference of those motivated to engage actively in interventional trials to address MCI. No attempt is made in the present analyses to suggest this is a representative sample of all persons with MCI or their caregivers.

\section{CONCLUSION}

The present findings provide preliminary support for the importance of QoL and self-efficacy outcomes to patients with MCI and their caregivers. In addition, the findings 
provide preliminary evidence that these preferences are fairly stable even after behavioral intervention. If these findings are supported by future research, they can provide valuable information for the design and focus of programs intended to meet the needs of patient and caregivers impacted by MCI.

\section{ACKNOWLEDGMENTS}

Research reported in this manuscript was partially funded through a Patient-Centered Outcomes Research Institute (PCORI) Award (CER-1306-01897). No funding was received for the publication of this article. The statements in this publication are solely the responsibility of the authors and do not necessarily represent the views of the Patient-Centered Outcomes Research Institute (PCORI), its Board of Governors or Methodology Committee.

All named authors meet the International Committee of Medical Journal Editors (ICMJE) criteria for authorship for this manuscript, take responsibility for the integrity of the work as a whole, and have given final approval for the version to be published.

Funding. Research reported in this manuscript was partially funded through a Patient-Centered Outcomes Research Institute (PCORI) Award (CER-1306-01897).

Disclosures. Polaris González Barrios, Ricardo Pabón González, Sherrie M. Hanna, Angela M. Lunde, Julie A. Fields, Dona E.C. Locke, and Glenn E. Smith have nothing to disclose.

Compliance with Ethics Guidelines. These studies were approved by the Mayo Clinic
Institutional Review Board. All procedures followed were in accordance with the ethical standards of the responsible committee on human experimentation (institutional and national) and with the Helsinki Declaration of 1964, as revised in 2013. Informed consent was obtained from all patients for being included in the study.

Open Access. This article is distributed under the terms of the Creative Commons Attribution-NonCommercial 4.0 International License (http://creativecommons.org/licenses/ by-nc/4.0/), which permits any noncommercial use, distribution, and reproduction in any medium, provided you give appropriate credit to the original author(s) and the source, provide a link to the Creative Commons license, and indicate if changes were made.

\section{REFERENCES}

1. Patient centered research institute, patient centered outcomes research. 2013 November 7, 2013 [cited 2016 April 15]; http://www.pcori.org/researchresults/patient-centered-outcomes-research.

2. Wadekar M, Sharma A, Battaglia G. Patient-centered outcomes research (PCOR): how can we optimize outcomes in cns research? Innov Clin Neurosci. 2015;12(3-4):27-31.

3. Institute of Medicine, Living well with chronic illness: a call for public health action. http://www. nationalacademies.org/hmd/ /media/Files/Report Files/2012/Living-Well-with-Chronic-Illness/living well_chronicillness_reportbrief.pdf National Academy of Sciences. Washington DC; (2012).

4. Riepe MW, et al. Quality of life as an outcome in Alzheimer's disease and other dementias-obstacles and goals. BMC Neurol. 2009;9:47-47.

5. Okonkwo $\mathrm{O}$, et al. Medical decision-making capacity in patients with mild cognitive impairment. Neurology. 2007;69(15):1528-35.

6. Albert $M$, et al. The diagnosis of mild cognitive impairment due to Alzheimer's disease: recommendations from the National Institute on 
Aging-Alzheimer's Association workgroups on diagnostic guidelines for Alzheimer's disease. Alzheimer's Dement J Alzheimer's Assoc. 2011;7(3):270-9.

7. Petersen $\mathrm{R}$, et al. Mild cognitive impairment: clinical characterization and outcome. Arch Neurol. 1999;56(3):303-8.

8. Smith G, et al. Definition, course and outcome of mild cognitive impairment. Aging Neuropsychol Cognit. 1996;3:141-7.

9. American Psychiatric Association. Diagnostic and Statistical Manual of Mental Disorders. 5th ed. Washington, DC: American Psychiatric Association; 2013.

10. Mayo Clinic, HABIT Health Action to Benenfit Independence \& Thinking. 20162016 4/1/2016]; Available from: http://www.mayo.edu/pmts/ mc2800-mc2899/mc2815-10.pdf.

11. Farias ST, et al. The measurement of everyday cognition (ECog): scale development and psychometric properties. Neuropsychology. 2008;22(4):531-44.

12. Berger $G$, et al. Longitudinal study on the relationship between symptomatology of dementia and levels of subjective burden and depression among family caregivers in memory clinic patients. J Geriatr Psychiatry Neurol. 2005;18(3):119-28.

13. Mattis S. Mattis dementia rating scale (MDRS). Odessa, FL: Psychological Assessment Resources; 1988.

14. Mittelman MS, et al. A three-country randomized controlled trial of a psychosocial intervention for caregivers combined with pharmacological treatment for patients with Alzheimer disease: effects on caregiver depression. Am J Geriatr Psychiatry. 2008;16(11):893-904.
15. Ayalon L, et al. Preferences for end-of-life treatment: concordance between older adults with dementia or mild cognitive impairment and their spouses. Int Psychogeriatr. 2012;24(11):1798-804.

16. Radloff LS. The CES-D scale: a self report depression scale for research in the general population. Appl Psychol Meas. 1977;1:385-401.

17. Logsdon RG, et al. Assessing quality of life in older adults with cognitive impairment. Psychosom Med. 2002;64(3):510-9.

18. Lorig KSA, Ritter P, Gonzalez V, Laurent D, Lynch J. Outcome measures for health education and other health care interventions. Thousand Oaks (CA: Sage; 1996.

19. Wisniewski SR, et al. The resources for enhancing alzheimer's caregiver health (reach): project design and baseline characteristics. Psychol Aging. 2003;18(3):375-84.

20. Galasko D, et al. ADCS prevention instrument project: assessment of instrumental activities of daily living for community-dwelling elderly individuals in dementia prevention clinical trials. Alzheimer Dis Assoc Disord. 2006;20(4 Suppl 3):S152-69.

21. Cummings JL, et al. The Neuropsychiatric Inventory: comprehensive assessment of psychopathology in dementia. Neurology. 1994;44(12):2308-14.

22. Bedard $\mathrm{M}$, et al. The Zarit burden interview: a new short version and screening version. Gerontologist. 2001;41(5):652-7.

23. Pearlin LI, et al. Caregiving and the stress process: an overview of concepts and their measures. Gerontologist. 1990;30(5):583-94. 